\title{
IMPACTO DE UN PROGRAMA DE PROMOCIÓN DE HIGIENE DE LA LENGUA EN ADULTOS MAYORES INSTITUCIONALIZADOS DE BUCARAMANGA*
}

\author{
${ }^{1}$ Yury Andrea Gómez, ${ }^{1}$ Angela Junca P., ${ }^{1}$ Diana Zulay Gutiérrez, ${ }^{1}$ Lyda Salazar Á., \\ ${ }^{2}$ Sonia Constanza Concha Sánchez \\ ${ }^{1}$ Estudiante de X semestre F. de Odontología U. Santo Tomás \\ ${ }^{2}$ Odontóloga U. Santo Tomás, Especialista en Educación y Comunicación para la Salud, MSc Epidemiología \\ U. Industrial de Santander, Docente U. Santo Tomás.
}

Autor responsable de correspondencia: Sonia Constanza Concha S.

Correo electrónico: sococosa@yahoo.com

\begin{abstract}
RESUMEN
Objetivo: Evaluar el impacto de un programa orientado a mejorar la higiene de la lengua en adultos mayores vinculados a dos centros geriátricos de Bucaramanga, desarrollado en el segundo semestre del 2006.

Materiales y métodos: Se realizó un ensayo clínico controlado. La muestra estuvo integrada por 50 personas, mayores de 50 años vinculados a dos instituciones geriátricas, 24 del grupo control y 26 grupo intervenido asignados aleatoriamente. Se recolectaron variables sociodemográficas, aspectos relacionados con higiene oral, índice cualitativo y cuantitativo de cobertura de la lengua antes y después del programa. Estas variables fueron comparadas según el grupo intervenido y no intervenido, para las variables cualitativas se aplicaron test de $\mathrm{Chi}^{2}$ y Exacto de Fischer y para la cuantitativas prueba t de Student y test de rangos de Wilcoxon, se consideró para el análisis un nivel de significancia $\alpha 0.05$.

Resultados: En la evaluación cuantitativa del nivel de higiene de lengua, después de la intervención, entre los grupos intervenido y no intervenido se evidencian diferencias estadísticamente significativas $(p<0.0001)$ con una media de 65.6 en el intervenido comparado con el no intervenido que registró un promedio de 135.7. La evaluación cualitativa del nivel de higiene de lengua posterior al desarrollo del programa, también permite evidenciar asociaciones estadísticamente significativas $(\mathrm{p}=0.001)$. Del total de personas con cobertura gruesa el 95\% (20) eran del grupo no intervenido, mientras que el 5\% (1) pertenecían al intervenido

Conclusión: La implementación de un programa de promoción de higiene en lengua en los adultos mayores reduce la cobertura de la capa de la lengua en los pacientes intervenidos comparado con los no intervenidos. [Gómez YA, Junca A, Gutiérrez DZ, Salazar L, Concha SC. Impacto de un programa de promoción de higiene de la lengua en adultos mayores institucionalizados de Bucaramanga. Ustasalud 2008; 7: 117 - 124]
\end{abstract}

Palabras clave: Lengua, Adulto mayor, Higiene oral, Índice.

IMPACT OF A TONGUE HYGIENE PROGRAM IN THE INSTITUCIONAL ELDERLY FROM BUCARAMANGA

\begin{abstract}
Purpose: To evaluate the impact of a tongue hygiene program in institutional elderly of Bucaramanga.

Materials and methods: A randomized clinical trial study was done. The sample was integrated by 50 adults, The control group: twenty four older. Intervention group: 26 elderly, assigned randomly. Sociodemographic variables, tissues related to oral hygiene, qualitative and quantitative tongue hygienic before and after the program were collected. By statistical analysis these variables were compared according intervention group, for the qualitative variables Chi square and Fischer Exact test and for the quantitative $t$ Student and Wilcoxon rank test were applied, level of significance to 0.05 .

Results: Between control and intervention group statistically significant differences were observed. Quantitative index the means of oral hygienic index in the intervention group was 65.6 and in the control 135.7. The qualitative evaluation of the level of tongue hygiene, also allows to demonstrate statistically significant associations $(\mathrm{p}=0.001)$. Of the total of people with heavy cover, $95 \%(20)$ were in no intervention group and $5 \%$ to intervention group.

Conclusion: The implementation of a tongue hygienic program in the elderly reduces the cover of the layer of the language in the intervention group compared with no intervention group.
\end{abstract}

Key words: Tongue, Elderly, Oral hygienic, Index.

Recibido para publicación: 29 de septiembre de 2008. Aceptado para publicación: 14 de diciembre de 2009.

* Grupo de Investigación SYBAM 


\section{INTRODUCCIÓN}

El interés de todo profesional de la salud es el de brindar a sus pacientes alternativas que les permitan conservar y mejorar su salud. Los odontólogos no pueden permanecer ajenos a esta realidad pues, además de constituirse en actividades inherentes a su ejercicio; tienen el compromiso ético de proveer las mejores alternativas de atención a sus pacientes, fundamentados en la mejor evidencia. ${ }^{1,2}$

La adopción de medidas de autocuidado y particularmente de higiene oral han demostrado ser las mejores alternativas para orientar acciones tanto de promoción como de prevención a nivel bucodental; sin embargo, a pesar del impacto que han generado, no han alcanzado el nivel deseado y esto puede deberse al hecho que existen grupos particulares que requieren de medidas de higiene oral especiales acordes con sus necesidades. ${ }^{3,4}$

Las personas adultas mayores registran graves problemas orales y aunque no son muy claras las causas relacionadas con este fenómeno; si se ha hecho evidente que son múltiples los factores que pueden incidir, dentro de las que cabría considerar una pobre higiene oral. ${ }^{4-7}$

La higiene oral en los adultos mayores no debe circunscribirse al mantenimiento de una buena higiene de los dientes, debe involucrar también la higiene de las prótesis dentales y de la lengua, pues se constituyen estructuras sobre las cuales puede depositarse la placa dentobacteriana y crear un ambiente para el desarrollo de patologías orales, ${ }^{4,8}$ así como también, propiciar el crecimiento microbiano, particularmente de aquellos microorganismos catalogados como oportunistas; más aún, ser un medio que permita la proliferación de especies microbianas, que sin ser residentes normales, son transitorias pero con potencial patogénico a nivel sistémico. ${ }^{9}$

Sin embargo, el interés de promover la higiene de lengua no ha sido suficientemente difundido por el gremio odontológico y son muchos los adultos mayores que no han recibido instrucción mínima en higiene oral y particularmente de la lengua. ${ }^{9,10}$

Al considerar las potenciales falencias en el proceso educativo y las posibles repercusiones sobre la condición general y oral, se hace necesario implementar programas orientados a fortalecer este hábito de autocuidado.

Lo ya expuesto, llevó a la realización de este trabajo, cuyo objetivo fue evaluar el impacto de un programa orientado a mejorar la higiene en len- gua en adultos mayores institucionalizados en el segundo semestre del 2006.

\section{MATERIALES Y MÉTODOS}

Se realizó un ensayo clínico controlado, ${ }^{11}$ donde 50 sujetos se eligieron mediante asignación aleatoria por conglomerados a ser grupo intervenido conformado por 26 sujetos vinculados al Centro de Bienestar del Anciano Juan Pablo II de Floridablanca y otro grupo no intervenido conformado por 24 sujetos pertenecientes al Centro de Bienestar del Anciano de Bucaramanga. ${ }^{12}$

El universo lo conformaron 123 adultos mayores, institucionalizados de ambos géneros mayores de 50 años pertenecientes a los centros institucionalizados antes mencionados.

Los criterios de inclusión que se tuvieron en cuenta fueron: ser adulto mayor vinculado a alguna de las instituciones objeto del trabajo durante el año 2006 , mayores de 50 años. Se excluyeron del estudio aquellos adultos mayores con diagnóstico de patología siquiátrica mayor, alteraciones visuales severas y con enfermedades que dificultan sus labores de auto cuidado.

El tamaño de la muestra fue calculado mediante rutina simple size del programa EPI- INFO V6.04, ${ }^{13}$ con base en las siguientes especificaciones de diseño: prevalecia de mala higiene oral del grupo control del $75 \%$ y en el grupo intervenido del $25 \%$ en la prueba piloto, se consideró un nivel de confianza del $97.5 \%$ y un poder del $90 \%$ y una relación de expuesto y no expuesto de 1:1, lo que permite estimar que una muestra de 52 individuos, permitirán detectar diferencias estadísticamente significativas.

En este estudio se aplicó un instrumento para adultos mayores tipo encuesta diligenciada por un evaluador previamente entrenado y se recopilaron variables sociodemográficas como: edad, sexo, procedencia, estrato, vínculo institucional, régimen de seguridad social, tiempo vivido en el hogar, y variables clínicas como el índice cualitativo y cuantitativo de cobertura de la lengua antes y después del programa planteado por Miyazaki; ${ }^{14}$ intervención, nivel de enseñanza relacionado con la limpieza de la lengua y la variable: cepillar la lengua produce náuseas.

Con la finalidad de evaluar el instrumento utilizado para la recolección de la información, se efectuó una prueba piloto que permitió mejorar la calidad, precisión y pertinencia de las preguntas, así como estandarizar los procedimientos de diagnóstico. La prueba se realizó en adultos mayores pertenecien- 
tes al barrio La Paz de Floridablanca, diferentes a los adultos mayores participantes en el estudio y con características semejantes a la población objeto de estudio.

Para la recolección de la información se coordinó con los directores de las instituciones, se informó los objetivos del estudio y se solicitó el consentimiento informado de los participantes.

Para la recolección de la información relacionada con la higiene de lengua en adultos mayores, se les realizó el examen para observar el estado en que se encontraba las condiciones de higiene en la lengua antes y después de la intervención, para ello se utilizaron guantes, tapabocas, y un lugar iluminado y adecuado para mejorar la visibilidad de las superficies de la lengua.

Se realizó la modificación del método de Miyazaki, en el cual la lengua se dividió en segmentos; para la variable cualitativa se dieron valores de 0 a 2 , donde $0=$ sin cobertura, $1=$ cobertura delgada, $2=$ cobertura gruesa, posteriormente se tomó en cuenta el cuadrante que presentó mayor cobertura. ${ }^{14}$ Para la variable cuantitativa, se sumaron los valores de cada segmento, se dividieron en 9 y se multiplicó por 100. Posterior al examen oral se proporcionaron elementos de higiene oral a todos los adultos mayores de las dos instituciones.

Grupo no intervenido (Centro de Bienestar del Anciano de Bucaramanga): se les suministró los elementos de higiene oral y se les informó que debían realizar su higiene oral como normalmente lo hacían. .

Grupo intervenido (Centro de Bienestar del Anciano Juan Pablo II de Floridablanca): se ejecutó una estrategia especial diseñada por los investigadores que consistía :

- Fase I: Recolección de datos y toma de muestra.

- Fase II: Charla educativa en la que se aplicaron contenidos teóricos y prácticos.

- Fase III: Motivación, cepillado supervisado, en el cual se empleó la técnica de barrido, la cual consistía en cepillar el dorso de la lengua desde la parte posterior hacia el tercio anterior, hasta observar disminución en la cobertura de la lengua, y realizar la limpieza de su lengua diariamente, además se utilizó material educativo (rotafolio).

- Fase IV: Refuerzo de la información con macromodelo didáctico y cepillo dental y cepillado supervisado.

- Fase V: Cepillado supervisado y monitoreo.

- Fase VI: Cierre del programa integrado con un plegable con el objetivo de motivar al adulto ma- yor a que realice una correcta higiene de lengua y así reducir el índice de cobertura de la misma.

Una vez recolectada la información se procedió a efectuar la codificación de cada uno de los cuestionarios y a la sistematización por duplicado, en el programa Excel, ${ }^{15}$ la información obtenida se exportó al programa EPI-INFO $6.04^{13}$ y mediante la rutina validate se procedió a verificar la calidad de la digitación de la misma, se efectuaron las correcciones necesarias y se exportó al paquete estadístico STATA $9.0^{16}$ para su correspondiente procesamiento y análisis.

El plan de análisis univariado implicó el cálculo de medidas de resumen según la naturaleza de las variables. Para el bivariado se evaluaron asociaciones de la variable salida (intervenido) con cada una de las variables explicatorias mediante test de Chi Cuadrado y test Exacto de Fisher cuando las variables eran cualitativas y $t$ de student o test de rangos de Wilcoxon (según la distribución de los datos) cuando eran de naturaleza cuantitativa. Para el análisis se consideró un nivel de significancia de $\alpha=0.05$. 17,18

El estudio se realizó dentro de los principios éticos establecidos en la resolución 008430 del 4 de octubre de 1993 del Ministerio de Protección Social de la República de Colombia y en los que se preservaron los principios de autonomía, beneficencia, justicia, privacidad y confidencialidad frente a la información aportada. ${ }^{19}$

\section{RESULTADOS}

\section{Variables sociodemográficas:}

Se evaluaron un total de 50 personas de ambos géneros con un promedio de edad de $73.1 \pm 12.6$ años; $54 \%$ (27) eran hombres, el 47\% (23) son analfabetas, el 52\% (26) son del procedencia rural, el 94\% (47) pertenecen al estrato 1, el 86\% (43) están vinculados al régimen subsidiado y el promedio de tiempo que han vivido en el hogar fue de 8.4 \pm 8.2. La única variable en la que se observó diferencia estadísticamente significativa fue en la edad, $(\mathrm{P}=0.001)$ siendo mayor en el grupo intervenido. Pues mientras en el grupo intervenido la edad fue de $80.4 \pm 7.7$ años, en el no intervenido fue $65.1 \pm 2.2$ años (Tabla 1 ).

\section{Variables relacionadas con condición de salud general y hábitos:}

El 72\% (36) de las personas no presentan hábitos; el 54\% (27) reportaron no tener alteración sistémica, y el 78\% (39) ningún trastorno visual. Al analizar la 
variable hábitos, se encontraron asociaciones estadísticamente significativas para hábitos $(p=0.003)$, observándose que del total de personas que fumaban el $80 \%$ (8) pertenecían al grupo no intervenido y el $20 \%$ (2) al intervenido (Tabla 2).

\section{Variables relacionadas con higiene oral:}

Del total de la población el 54\% (27) no ha recibido enseñanza de cepillado, el 38\% (19) de las personas realiza su limpieza oral tres veces al día, el 80\% (40) reportó que al hacer la limpieza oral diaria se cepilla la legua; el 66\% (33) de la población informó que el cepillado de la lengua le produce náuseas, el $66 \%$ (33) realiza el cepillado de forma suave, el 90\% (45) no han presentado dolor en la cavidad oral en los últimos seis meses. No se observaron asociaciones estadísticamente significativas para ninguna de estas variables (Tabla 3).

Tabla 1. Descripción de las variables sociodemográficas de las personas mayores vinculados a instituciones geriátricas durante el II semestre del 2006 analizadas en forma global y según intervención.

\begin{tabular}{|c|c|c|c|c|}
\hline VARIABLES & $\begin{array}{l}\text { GLOBAL } \\
\text { FREC (\%) }\end{array}$ & $\begin{array}{l}\text { NO INTERVENIDO FREC } \\
(\%)\end{array}$ & $\begin{array}{l}\text { INTERVENIDO } \\
\text { FREC (\%) }\end{array}$ & $\mathbf{P}^{*}$ \\
\hline Global & $50(100)$ & $24(48)$ & $26(52)$ & \\
\hline Edad & $73.1 \pm 12.6$ & $65.1 \pm 12.2$ & $80.4 \pm 7.7$ & $0.001^{* *}$ \\
\hline \multicolumn{5}{|l|}{ Género } \\
\hline Hombre & $27(54)$ & $14(51.9)$ & $13(48.1)$ & $0.555^{* * *}$ \\
\hline Mujer & $23(46)$ & $10(43.5)$ & $13(56.5)$ & \\
\hline \multicolumn{5}{|l|}{ Nivel educativo } \\
\hline Ninguno & $23(47)$ & $10(43.4)$ & $13(56.6)$ & 0.712 \\
\hline Primaria & $20(41)$ & $10(50)$ & $10(50)$ & \\
\hline Secundaria & $4(8.2)$ & $3(75)$ & $1(25)$ & \\
\hline Universitario & $2(4.1)$ & $1(50)$ & $1(50)$ & \\
\hline \multicolumn{5}{|l|}{ Procedencia } \\
\hline Rural & $26(52)$ & $14(54)$ & $12(46)$ & $0.389^{* * *}$ \\
\hline Urbana & $24(48)$ & $10(42)$ & $14(58)$ & \\
\hline \multicolumn{5}{|l|}{ Estrato socioeconómico } \\
\hline Estrato 1 & $47(94)$ & $22(47)$ & $25(53)$ & \\
\hline Estrato 2 & $1(2)$ & $1(100)$ & $0(0)$ & 0.573 \\
\hline Estrato 3 & $2(4)$ & $1(50)$ & $1(50)$ & \\
\hline \multicolumn{5}{|l|}{ Régimen de salud } \\
\hline Ninguno & $2(4)$ & $0(0.00)$ & $2(100 \%)$ & 2.067 \\
\hline Subsidiado & $43(86)$ & $22(51.16)$ & $21(49 \%)$ & \\
\hline Contributivo & $4(8)$ & $1(25)$ & $3(75 \%)$ & \\
\hline No sabe & $1(2)$ & $1(100)$ & $0(0.00 \%)$ & \\
\hline Tiempo que ha vivido en este hogar & $8.4 \pm(8.2)$ & $12.3 \pm 10.08$ & $4.8 \pm 3.37$ & \\
\hline
\end{tabular}

${ }^{*}$ Test exacto de Fischer ${ }^{* *}$ test de Student ${ }^{* * *} \operatorname{chi}^{2} \alpha=0.05$ 
Tabla 2. Descripción de variables relacionadas con la condición de salud general y los hábitos en los adultos mayores vinculados a instituciones geriátricas durante el II semestre de 2006 analizados en forma global y según la intervención.

\begin{tabular}{lcccc}
\hline VARIABLE & $\begin{array}{c}\text { GLOBAL } \\
\mathbf{n}=\mathbf{5 0} \\
\text { FREC (\%) }\end{array}$ & $\begin{array}{c}\text { NO INTERVENIDO } \\
\mathbf{n = 2 4} \\
\text { FREC (\%) }\end{array}$ & $\begin{array}{c}\text { INTERVENIDO } \\
\mathbf{n = 2 6} \\
\text { FREC (\%) }\end{array}$ & P* $^{*}$ \\
\hline $\begin{array}{l}\text { Hábitos } \\
\text { Fumar }\end{array}$ & $10(20)$ & $8(80)$ & $2(20)$ & $0.003^{* *}$ \\
Alcohol & $4(8)$ & $4(100)$ & $0(0)$ & \\
Ninguno & $36(72)$ & $12(33.3)$ & $24(67.6)$ & \\
Enfermedad o alteración sistémica & & & & \\
No & $27(54)$ & $13(48)$ & $14(52)$ & $0.565^{*}$ \\
Si & $22(44)$ & $10(45)$ & $12(55)$ & \\
No sabe & $1(2)$ & $1(100)$ & $0(0)$ & \\
Transtorno visual & & & & \\
No & $39(78)$ & $20(51)$ & $7(49)$ & $0.382^{* *}$ \\
Si & $11(22)$ & $4(36)$ & $7(64)$ & \\
\hline
\end{tabular}

${ }^{*} \mathrm{Chi}^{2}{ }^{* *}$ test exacto de Fischer $\alpha=0.05$

Tabla 3. Descripción de las variables relacionadas con la higiene oral en adultos mayores vinculados a instituciones geriátricas durante el II semestre de 2006 analizados en forma global y según intervención.

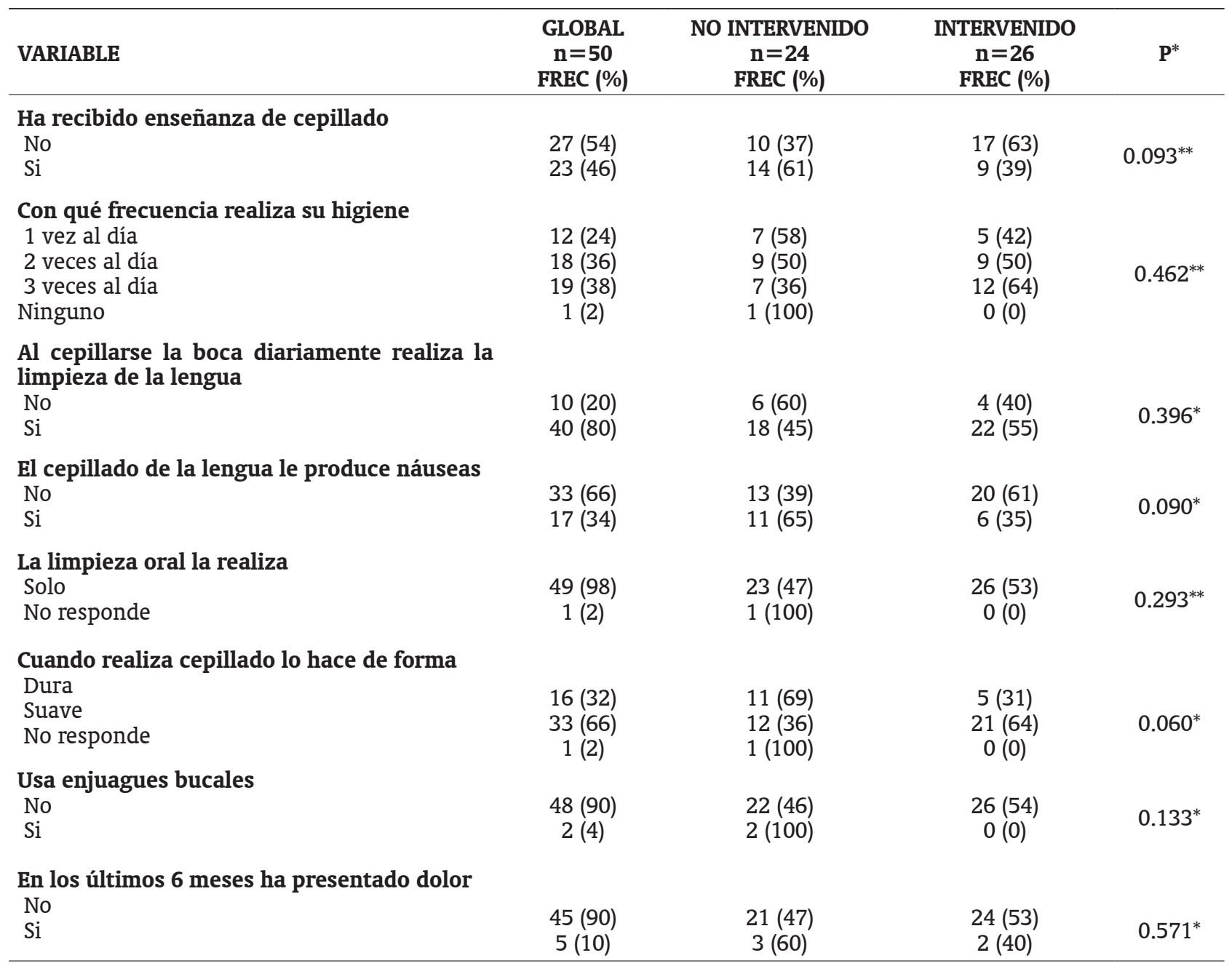

${ }^{*} \mathrm{Chi}^{2}{ }^{* *}$ test exacto de Fischer 


\section{Variables relacionadas con higiene de lengua:}

Antes de la intervención se observaron diferencias estadísticamente significativas $(\mathrm{p}=0.001)$ para el nivel de higiene de lengua analizado en forma cuantitativa siendo menor en el grupo que iba a ser intervenido. Posterior a la intervención se observó una reducción del conteo de placa en lengua casi a la mitad en el grupo intervenido si se compara con el antes de este mismo grupo. Adicionalmente, al comparar el promedio de conteo de placa después de la intervención entre los grupos intervenido y no intervenido se evidencian diferencias estadísticamente significativas $(\mathrm{p}<0.0001)$ con una media de 65.6 en el intervenido comparado con el no intervenido que registró un promedio de 135.7.

La evaluación cualitativa del nivel de higiene de lengua posterior al desarrollo del programa, también permite evidenciar asociaciones estadísticamente significativas $(\mathrm{p}=0.001)$. Del total de personas con cobertura gruesa el 95\% (20) eran del grupo no intervenido, mientras que el $5 \%$ (1) pertenecían al intervenido (Tabla 4).

Tabla 4. Descripción de las variables relacionadas con el nivel de higiene de lengua en adultos mayores vinculados a instituciones geriátricas durante el II semestre de 2006 analizadas en forma global y según intervención.

\begin{tabular}{|c|c|c|c|c|}
\hline VARIABLE & $\begin{array}{c}\text { GLOBAL } \\
n=50 \\
\text { FREC (\%) }\end{array}$ & $\begin{array}{c}\text { NO INTERVENIDO } \\
n=24 \\
\text { FREC (\%) }\end{array}$ & $\begin{array}{c}\text { INTERVENIDO } \\
\mathbf{n}=\mathbf{2 6} \\
\text { FREC (\%) }\end{array}$ & $\mathbf{P}^{*}$ \\
\hline \multicolumn{5}{|l|}{ Cualitativa antes } \\
\hline Cobertura delgada & $6(12)$ & $1(17)$ & $5(83)$ & 0.101 \\
\hline Cobertura gruesa & $44(88)$ & $23(52)$ & $21(48)$ & \\
\hline \multicolumn{5}{|l|}{ Cualitativa despues } \\
\hline Sin cobertura & $1(2)$ & $0(0)$ & $1(100)$ & \\
\hline Cobertura delgada & $27(55)$ & $3(11)$ & $24(89)$ & \\
\hline Cobertura gruesa & $21(43)$ & $20(95)$ & $1(5)$ & 0.0001 \\
\hline \multicolumn{5}{|l|}{ Cuantitativa } \\
\hline Antes & $138.3 \pm 37.5$ & $155.3 \pm 29.4$ & $122.6 \pm 37.8$ & $0.0001^{*}$ \\
\hline Después & $98.5 \pm 50.49$ & $135.7 \pm 45.2$ & $65.6 \pm 26.3$ & $<0.0001^{* *}$ \\
\hline
\end{tabular}

${ }^{*}$ Test exacto de Fischer ${ }^{* *}$ test de Student

\section{DISCUSIÓN}

Según el Estudio Nacional de Salud Bucal (ENSAB III), se ha observado una lenta pero progresiva recuperación de la salud bucal en los últimos años. A pesar de lo anterior, el deterioro de la condición bucodental se produce rápidamente con el paso de la adolescencia a la edad adulta, dado que el porcentaje de dientes sanos se reduce al 50\%. ${ }^{5}$

Lo anterior ilustra sobre la necesidad de implementar, evaluar y dar continuidad a los programas promocionales y preventivos ( $\mathrm{P}$ y $\mathrm{P}$ ) dirigidos a las personas adultas y muy especialmente a las personas adultas mayores. Pero este tipo de acciones deben incluir el control de todos los factores de riesgo potencial que pueden afectar a este grupo de edad dentro de los que cabría considerar la placa dentobacteriana. ${ }^{4}$

Los odontólogos no deben olvidar que la acumulación de este depósito microbiano puede ocurrir en cualquier superficie dura (prótesis dentales, dien- tes) o blanda (mucosa de lengua y carrillos, entre otros ) y puede llegar a desencadenar enfermedades a nivel local o general. ${ }^{4,9}$

Al tener en cuenta los aspectos anteriormente mencionados, el objetivo principal de todo programa de $P$ y $P$ dirigido a adultos mayores debe estar orientado a mejorar la calidad de vida del adulto mayor, para, con este tipo de acciones, reducir la presencia de enfermedades que pueden ser prevenibles o controladas, incentivar mejores alternativas para las personas mayores que pueden verse afectadas por un buen número de alteraciones, físicas o mentales. ${ }^{20}$

El objetivo del presente trabajo fue evaluar el impacto de un programa de promoción de la salud oral y prevención de las patologías orales y que estaba orientado a mejorar la higiene de lengua en adultos mayores institucionalizados del Centro de Bienestar del Anciano de Bucaramanga y del Centro de Bienestar del Anciano Juan Pablo II de Floridablanca que se desarrolló en el segundo semestre del 2006. 
Una experiencia anterior, desarrollada por el grupo de Salud Integral y Bienestar del Adulto Mayor (SIBAM), mostró cómo la implementación de este tipo de acciones tiene un buen efecto en la reducción de la placa dentobacteriana a nivel de estructuras dentales y protésicas; sin embargo, no fue tan efectivo en lograr cambios relacionados con el mejoramiento de la higiene de lengua. El grupo que desarrolló la actividad argumentaba como potencial causa de estos resultados al hecho de que las personas mayores registraban náuseas y esto limitaba que los sujetos intervenidos cumplieran con el cepillado de la lengua en forma adecuada. ${ }^{21}$ Sin embargo, estudios posteriores parecían indicar que la dificultad se centraba más en problemas que registraban los operadores a la hora de manejar el índice de higiene de lengua. ${ }^{22}$ Esto motivó a desarrollar un proceso investigativo en el que se perfeccionó el manejo del índice y verificó su potencialidad como medio de diagnóstico. ${ }^{23}$

Dentro de los procesos desarrollados se incluyó la definición de los procedimientos para la aplicación del índice, y estandarización de los evaluadores para el manejo tanto cualitativo como cuantitativo de este indicador, lográndose niveles de reproduciblidad para el índice analizado en forma cualitativa de 0.70 y para el cuantitativo 0.83 . El primero analizado mediante Kappa ponderado y el segundo mediante coeficiente de correlación de rangos de Sperman. Por lo cual la evaluación de la intervención se pudo verificar fundamentados en un índice al que se le había analizado su potencialidad como medio diagnóstico. ${ }^{23}$

Adicionalmente, estudios realizados por Mantilla y colaboradores plantearon una modificación del índice de Miyazaki, para investigar la prevalencia de la cobertura de la lengua en sujetos sanos, con gingivitis y en pacientes con periodontitis, para determinar la relación entre cobertura de lengua y carga bacteriana en muestras salivares. En conclusión, el índice desarrollado y que se aplicó en este trabajo, indicó que la mayoría de coloración y cobertura está localizada en la parte posterior especialmente en la sección media, se encontró una relación entre la edad y la presencia de una apariencia con cobertura. Aspecto que fue similar al observado en el presente trabajo, pues al inicio del estudio el $88 \%$ de los adultos mayores evaluados registraron una cobertura gruesa en la lengua y usualmente ubicada en el la región posterior de la lengua. ${ }^{14}$

Es importante anotar que en el presente trabajo, el $54 \%$ de las personas mayores no recibieron enseñanza sobre la higiene de la lengua, resultados similares fueron reportados por Ballesteros y colaborado- res, ${ }^{23}$ quienes evidenciaron en su investigación que el 69\% de los pacientes afirmaron no haber recibido ninguna instrucción orientada a promover la higiene de lengua. Esto muestra la necesidad de implementar procesos en los que las actividades educativas orientadas con la higiene oral, particularmente de la lengua, se desarrollen en forma efectiva. Esta última afirmación se sustenta en los resultados de este trabajo pues se logró reducir a la mitad la cantidad de placa bacteriana de la lengua en el grupo que en el que se implementó este programa.

Se podría concluir que con este programa educativo se evidenciaron cambios en la higiene de lengua de los adultos mayores sólo después de realizada la intervención lo que demuestra que la implementación de programas educativos en adultos mayores son una buena estrategia preventiva para lograr un impacto a corto plazo en la higiene de lengua, Sin embargo, es importante reconocer la necesidad de darle continuidad a este tipo de programas si se quiere la apropiación del hábito por parte de los adultos mayores.

\section{BIBLIOGRAFÍA}

1. Howes F, Doyle J, Jackson N, Waters E. Evidence-based public health: the importance of finding "difficult to locate" public health and health promotion intervention studies for systematic reviews. J Public Health 2004; 26: $101-104$.

2. Tang KC, Ehsani JP, McQueen DV. Evidence based health promotion: recollection, reflections, and considerations. J Epidemiol Community Health 2003; 57: 841 - 843.

3. Jackson RJ, Newman HN, Smart GJ, Stokes E, Hogan JI, Brown C, Seres J. The effects of a supervised toothbrushing programme on the caries increment of primary school children, initially aged 5-6 years. Caries Res 2005; 39: $108-115$.

4. Concha SC. Importancia de los índices que evalúan la higiene oral de prótesis dentales removibles en la implementación de programas promocionales y preventivos y criterios básicos para evaluar su reproducibilidad. Ustasalud Odontología 2005; 4: 38 - 43.

5. República de Colombia. Ministerio de Salud. Estudio Nacional de Salud Bucal,1999

6. Petersen PE, Yamamoto T. Improving the oral health of older people: the approach of the WHO Global Oral Health Programme. Community Dent Oral Epidemiol 2005; 33: 81 - 92.

7. Bermúdez W, Concha SC, Camargo DM. Perfil orofacial de las personas mayores institucionalizadas de la ciudad de Bucaramanga y su área metropolitana. Ustasalud Odontología 2003; 2: 13 - 19.

8. Sheen SR, Harrison A. Assessment of plaque prevention on denture using an experimental cleanser. J Prosthet Dent 2000; 84: 597 - 601.

9. Ruiz-Medina P, Bravo M, Gil-Montoya JA, Montero J. Discrimination of functional capacity for oral hygiene in elderly Spanish people by the Barthel General Index. Community Dent Oral Epidemiol 2005; 33: 363 - 369. 
10. López C, Bejarano D, Jiménez DM, Mamián Z, Morales LF, Calzada MT, Barreto JF. Influencia de dos técnicas de higiene de lengua en el sentido del gusto en ancianos de cuatro hogares geriátricos de Cali. Memorias XVII Encuentro de Investigación ACFO. 2006

11. Blanco JH, Maya JM. Tomo III Epidemiología Básica y principios de Investigación. Primera Edición. Corporación para Investigaciones Biológicas. Medellín. 2000

12. Lohr SL. Muestreo: Diseño y análisis. México: Internacional Thomson Editores. 2000 p. $4-21$.

13. CDC-OMS. Epi-info 6, Versión 6.04. Atlanta, (Nov 1996)

14. Gomez SM, Danser MM, Sipos PM, Rowshani B, Van Der Velden U, van der weijden GA. Tongue coating and salivary bacterial counts in healthy/gingivitis subjects and periodontitis patients. J Clin Periodontol 2001; 28: 970 -978 .

15. Microsoft Excel 5.0. NY. Microsoft Corporation, 1997

16. Stata Corp. Stata Statistical Software. Release 9.0. College station. TX: Stata corporation 2005

17. Pagano $M$ and Gauvreau K. Principles of Bioestatistics. Belmont: Duxbury Press. 1995 p. 35-44, 257-263

18. Norman GR, Streiner DL. Bioestadística. Madrid:Mosby/ Doyma Libros, 1996. p. 129-142

19. República de Colombia. Ministerio de Salud. Resolución No. 008430 (Octubre 4 1993); Bogotá.

20. Magallon R. Diseño de una propuesta de intervención educativa en adultos mayores basándonos en los resultados de la Escala de Satisfacción Personal de Filadelfia en la Universidad La Salle. URL disponible en: http://comunidad.ulsa.edu.mx/public_html/editorial/editorialfull.php

21. García YM, Flórez LJ, Silva LM, Aguilar E, Concha SC. Evaluación de la efectividad de un programa educativo en higiene oral dirigido a cuidadores de adultos mayores de los asilos de San Antonio y San Rafael de la ciudad de Bucaramanga. Ustasalud Odontología 2006; 5: 40 - 48.

22. Gómez MA, Bernal MF, Rodríguez DA. Evaluación de un programa en salud oral dirigido a cuidadores de adultos mayores institucionalizados de los asilos San Antonio y San Rafael de Bucaramanga [Trabajo de grado]. Bucaramanga: Universidad Santo Tomás; 2006.

23. Ballesteros I, Cobo KA, Navarro JP, Concha SC. Evaluación de la reproducibilidad de dos índices orientados a analizar la higiene de la lengua. Ustasalud Odontología 2008; 7: $35-41$.

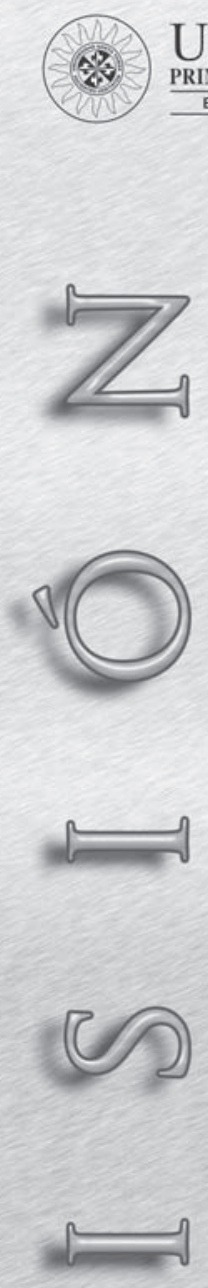

Universidad Santo Tomas PRIMER CLAUSTRO UNIVERSITARIO DE COLOMBIA \begin{tabular}{lllllllllll}
$B$ & $U$ & $C$ & $A$ & $R$ & $A$ & $M$ & $A$ & $N$ & $G$ & $A$ \\
\hline
\end{tabular} iHace Pais!

La Facultad de Odontologia de la Universidad Santo Tomás en concordancia con la visión institucional aspira lograr:

Un compromiso efectivo de la comprensión renovada del hombre colombiano, su mundo y sus valores, en un contexto de salud integral para todos; el reconocimiento nacional e internacional, como comunidad académica, a través de la investigación, la producción intelectual y el intercambio académico; liderazgo en los procesos relacionados con la salud bucal de la comunidad, a nivel regional y nacional; la cualificación permanente de sus servicios a la comunidad para responder efectiva y oportunamente a sus usuarios; el fortalecimiento permanente de su accionar pedagógico mediante el desarrollo humano integral, la aplicación de tecnologias de punta y la proyección comunitaria. 